\title{
Platelet rich plasma therapies: a great potential to be harnessed
}

Isabel Andia

Regenerative Medicine Laboratory, BioCruces Health Research Institute, Cruces University Hospital, Barakaldo, Spain

Platelet rich plasma (PRP) therapies are a sensitive and controversial topic in Orthopaedics and Sports Medicine. Since the pioneer work by Sanchez et al. ${ }^{1}$ in Sports Medicine, the use of PRPs has expanded to meet multiple medical problems where current treatment options were judged to be suboptimal. This rapid expansion has been possible given their safety profile, i.e. autologous source, and minimal manipulation. This issue of Muscles Ligaments and Tendons Journal gathers the work of basic and clinical researchers with multidirectional inputs, and highlights the relevance of translational research in producing the essential scientific knowledge to design successful therapies.

The use of PRP therapies started in the clinics rather than in the laboratories, and the rapidly evolving basic PRP knowledge questions the accuracy of PRP description introduced as MeSH (Medical Subject Headings) in 2007: "a preparation consisting of PLATELETS concentrated in a limited volume of $P L A S M A$. This is used in various surgical tissue regeneration procedures where the GROWTH FACTORS in the platelets enhance wound healing and regeneration". Indeed, new proteomic analyses of platelet secretome list several hundreds of proteins, and includes not only GROWTH FACTORS, but also a vast array of molecules including cytokines and chemokines, adhesive proteins, and enzymes: GROWTH FACTOR are in reality just a small subset ${ }^{2}$. PRP science is now so complex that devising an appropriate formulation requires further attention to the host tissue context. To tell the truth, PRP therapies are not always successful. Why some patients respond to PRP therapies while others do not; why the same athlete may heal his tendon in one occasion, and fails to heal another tendon in another occasion, without inter-procedural variations, remains an enigma.

It is possible that one PRP formulation is appropriate for certain conditions and not as suitable for others. And it is not only the formulation: the volume and protocol for application may be more important than the formulation per se.

Inter-individual differences in PRP quality as well as the conditions of the host tissue receiving the PRP may account for the varied outcomes. The differential response among patients would suggest that PRP therapies could be directed in an individualized manner to treat specific subgroups of patients. Thus, personalized medicine may be applied to the field of PRPs. Revealing crucial facts underlying the success or the failure of PRP is a current challenge for PRP researchers.

One could question the generalizability of PRP therapies, leading to an interpretation that the progression of tendinopathies and joint pathologies could be reduced, and even reversed, by PRP stimulation. High level of evidence studies are beginning to emerge and provide data for the management of tendon and osteoarticular conditions. Recently, a quantitative synthesis $^{3}$ and a meta-analysis ${ }^{4}$ in knee joint cartilage degenerative pathology concluded that PRP tends to be more effective than HA administration, with patients with lower degrees of degeneration benefiting more. However, mostly symptomatology is evaluated, and so far MRI data evaluating OA progression are lacking. Similar circumstances affect tendinopathy. Constant re-evaluation to identify protocols that are likely to succeed is important for a rapid development on the field.

PRP science is in its very beginning, and PRPs are used with limited mechanistic understanding of their molecular and cellular properties. Without knowledge concerning mechanisms of action, questions regarding dosage (platelet and leukocyte count and/or plasma volume), timing of delivery, and number of administrations are in its firsts.

Thus, there is a clear need to stratify patients, and augment our knowledge by introducing novel approaches such as combination products, as well as procedural modifications tailored to every single condition: this may help to control cell activities for successful healing. Over the next few years, we hope to harness the full potential of PRP for managing musculoskeletal problems.

For the time being, we have gathered under the same roof studies which promise to bridge this translational gap: enjoy.

\section{Acknowledgements}

I would like to thank everyone who helped make this special PRP issue of MLTJ.

A huge thank you goes out to Prof. Nicola Maffulli with whom I am proud to collaborate, and to Dr. Francesco Oliva for his commitment to MLTJ growth. 


\section{References}

1. Sánchez M, Azofra J, Anitua E, Andia I, et al. Plasma rich in growth factors to treat an articular cartilage avulsion: a case report. Med Sci Sports Exerc. 2003;35(10):16481652.

2. Andia I, Maffulli N. Platelet-rich plasma for managing pain and inflammation in osteoarthritis. Nat Rev Rheumatol. 2013;9 (12):721-730.
3. Khoshbin A, Leroux T, Wasserstein D, et al. The efficacy of platelet-rich plasma in the treatment of symptomatic knee osteoarthritis: a systematic review with quantitative synthesis. Arthroscopy. 2013;29(12):2037-2048.

4. Chang K-V, Hung Ch-Y, Aliwarga F, et al. Comparative effectiveness of platelet-rich plasma injections for treating knee joint cartilage degenerative pathology. A systematic review and meta-analysis. Arch Phys Med Rehabil. 2013. pii: S00039993(13)01212-4. doi: 10.1016/j.apmr.2013. 